\title{
Social Media, Dietetic Practice and Misinformation: A triangulation research
}

\author{
Dr. Simran Sidhu ${ }^{1}$
}

\begin{abstract}
Understanding the credibility of social media to produce reliable and factual content is crucial especially in the field of health communication. Social media carries information in multiple layers which is often supported with photographs, images, audio video clips of health experts/trainers, which is further not backed by any sort of certification. Blogs, twitter, Instagram and Facebook are few tools which keep regularly update and track the followers, members as peers on social media. Facebook attracts the maximum and could be considered as power house of such information. It is assumed that some of the groups loading daily dose of fitness might not exist without Facebook.

In the present study triangulation method of research is applied to evaluate the awareness and application of information related to diet for health, fitness and reduce body weight. To study interdependencies the research focused on content floated on social media and dietetic practice adopted by the respondents. The research had quantitative and qualitative approach. A survey was conducted by developing a questionnaire. The primary data was collected from 200 respondents of Jalandhar district. The study strives to examine the information on social media and its adaptation in routine life among different age groups.
\end{abstract}

Key-words: Health issues, Dietetic practices, Agenda building, discourse

\section{Introduction}

The reach of $4 \mathrm{G}$ high speed bandwidth has brought revolutionary changes in the concept of information flow. Further whether this information leads to upgrade knowledge and enhance the quality of life style of Indians is again a questionable issue. The social media is playing a significant role in generating and transferring information voluminously every day. Social media is flooded with unaccountable information related to politics, travel tourism, culture entertainment and health etc.

Mainstream media could not enrich us with such voluminous information related to health till now on contrary to one click on social media that leads to hundreds of self-glorious beautiful faces on Facebook and Instagram followed by healthy living and fitness bloggers. Pinterest has numerous knowledgeable audio video clips of fat burn, weight loss magic techniques which lure the netizens to click and dive into ocean of vast information. Making effective use of the information is sole responsibility of the surfer, for which awareness is very crucial or information could prove fatal.

Effective IEC (Information, Education and Communication) is the backbone of human development and the advent of social media has enhanced the flow of IEC. This platform is utilized for generation of information and distribution of information simultaneously.

1 Assistant Professor \& Head, P.G. Deptt. Of JMC, Doaba College, Jalandhar
Considering it as a two way communication, the networking communities of social media and open-access also create a threat whether the information is shared and used results in development or goes directionless. Sharing of information related to science and health is important only when health experts, patients or users of such information are constantly in touch.

Through blogs the health specialists and researchers convey about their latest research work, the government and public health department can inform or alarm the general public regarding various health issues, spread of a particular disease or infection (Dengue, Bird flu etc.) and preventive measures regarding it. Twitter keeps the sender and receiver in constant touch with number of follows. The advantage of these online discussion forms is that it allows people to connect over powering all geographical and institutional applications.

In India, though the number of netizens is ever increasing but the effective use of social media is still questionable. The field of health education and awareness also adapted to social media but how far it reaches amongst the public, had always been a matter of concern.

Health issues need to be mirrored comprehensively and proportionally. The social media content in context of health and healthy life had always been lacking accuracy and authenticity once uploaded, the information travels to millions of social media alcoholics before the verification of its facts and sources. Half cooked data with text images and audio 
visuals is followed blindly to grab eyeballs towards shaped up few figures. Crash dieting, protein intakes, gym-culture leads to a particular market to accomplish the desired results. Knowing least about the side effects and harm to the vital parts of the body in the later age, the users are trapped in the web of precision marketers.

The socio-cultural idealization of thinness or zero figures is another reason of eating disorders. Pinterest or Instagram are flooded with a number of rare fruits, seeds and plants that burn body fat at speed. These 'rare' plants and fruits images with captions assuring a loss of few kilograms in a week/15 days are a common scene on social media such information capsules with long list of comments as follow-ups further add up to personal reference on health.

\section{Objectives of the study}

- To study the use of social media for health and fitness information by general public

- To study the response and awareness level of the social media users to information related to health fitness

- To gauge the application of the health awareness information floated on social media

\section{Research Methodology}

The study has been conducted by applying triangulation method in which information was collected through primary and secondary data. The primary data was collected applying survey method. The respondents chosen were according to the purpose of the study. The research was done disseminating questionnaire in 200 respondents. These respondents were also virtually present on social media. Primary data was collected on priority as this study draws direct information from the field. A close ended questionnaire with 28 questions was disseminated to 200 respondents where Jalandhar district was the universe of the study. Further the respondents were equal in number i.e., 100 males and 100 females. The sampling technique used for the study was purposive sampling under non probability sampling technique. The qualitative study of health and fitness information on social media was conducted on Facebook groups, Instagram, Pinterest and blogs.

\section{Data Analysis and Inferences}

The population (total number of respondents) taken was 200, it was further divided equally among males and females. The respondents were both rural and urban where $20 \%$ of males and
$30 \%$ of females were rural. Demographically, $10 \%$ of the rural and $20 \%$ urban males fall in the age group of $20-25$ years as compared to $30 \%$ rural females and only $10 \%$ females belonged to urban area.

$40 \%$ of the males (urban) were of $25-30$ years as compared to $30 \%$ of the urban females of the same age. Equal \%age (10\%) of males both urban and rural fall in the age group of 30-35 years. There were $10 \%$ of urban females in this age group. $10 \%$ of urban males and $20 \%$ of urban females belonged to 35-40 years of age group.

a) Social media is a platform where information pops up at greater speed than any other medium. The number of social media users adds up enormously every single day. The research reveals that the concept of social media is yet to be understood by its users as only $45 \%$ of the total respondents know that social media includes all Facebook, Instagram, twitter, blogs, WhatsApp, Pinterest etc. This may be due to more users of Facebook and WhatsApp 55\% as compared to $30 \%$ who also use Instagram. Interestingly, $10 \%$ of the respondents only used Instagram as platform to share and exchange information on social media. Only $5 \%$ of the respondents between 20 to 40 years of age used or followed someone on twitter. The survey also reveals that in Jalandhar city, the social media users preferred WhatsApp 75\% followed by Instagram $15 \%$ and only $10 \%$ of the were frequently active on Facebook. As Facebook has a unique feature of making 'friends', evading all geographical boundaries. The respondents make friends; join groups and forums with or without knowing much about the hidden agendas, mission or approach of the latter.

b) Research shows that $60 \%$ of the social media users join/subscribe to groups/forums for information, education and entertainment. $25 \%$ of the respondents seek only information and also suggest others to join such forums. Interestingly $100 \%$ female respondents suggest others to join/subscribe groups. Thus female social media users make more recommendations indirectly divulging into marketing products/services/ideas knowingly/unknowingly the details. The respondents are not clear about the concept of two-way communication and $75 \%$ of the respondents considered it as group communication. Knowing least about the concept of two way communication, the respondents prefer more informal and personal interaction. Unaware about the 
concept that their queries are answered by the marketers expertise in opinion building. The social media sites promoting organic food, clean diet etc make sure that no question goes unanswered on such groups and forum which promote health fitness, zero figures and weight loss etc. The data reveals that only $15 \%$ of the total respondents refer a doctor. $20 \%$ males and $15 \%$ females sought expert advice from health trainers, where the health trainers are not expert on physiology and learn gym training to be health trainers. Only $5 \%$ visit dieticians to interact interpersonally for confirmation of their information and source of information. Thus social media is established platform for marketers to reach masses across the globe.

c) Facebook has the characteristics of two way communication where the respondents seek not only response to their quarries but also welcome valuable suggestions from 'friends' which may be or not from the field of health. The activity on Facebook is designed to attract attention. People on Facebook expect reactions for everything they float on Facebook. The marketers/companies know the potential of Facebook and make links through email/websites/YouTube with the users. Almost all respondents agree that health related information $(25 \%$ males $)$ regularly pop up while using Facebook whereas $40 \%$ respondents rarely came across such health fitness groups/forums as compared to $35 \%$ respondents sometimes come across such information while on Facebook.

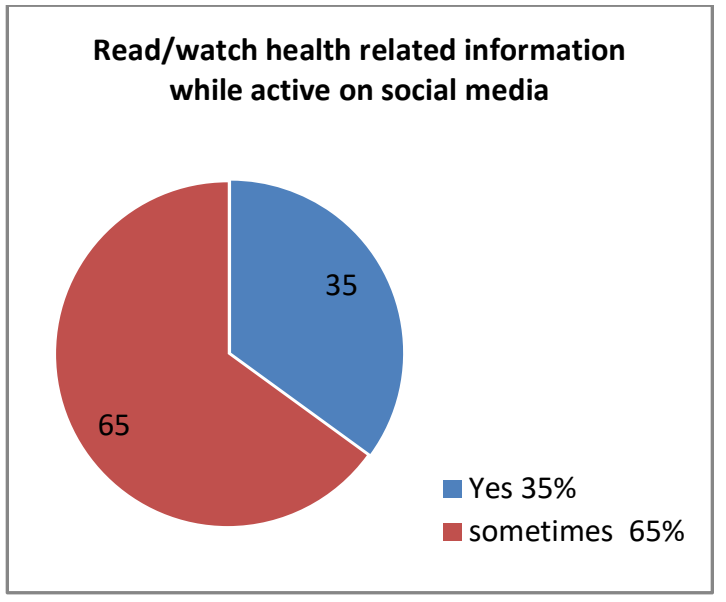

$65 \%$ of the respondents sometimes read/watch health related information while being active on social media as compared to $35 \%$ read it regularly though not regular but all 200 respondents read health related information on social media. 30\% female respondents as compared to $5 \%$ males regularly read/watch health related information.

d) Social media multiplies information manifolds simultaneously as the study reveals that $35 \%$ of the social media users read and watch health related information while active on social media whereas 65\% respondents also sometimes read it. Comprehensively the information on social media may it be images, texts, videos never goes unseen. The study shows that $40 \%$ of the males further reach out to read and seek more knowledge by clicking hyperlinks, only 15\% said 'no' and $45 \%$ of the total respondents sometimes click the hyperlinks. The Facebook users $(65 \%)$ promotes, discuss this information further with different groups/parents/friends and siblings etc.

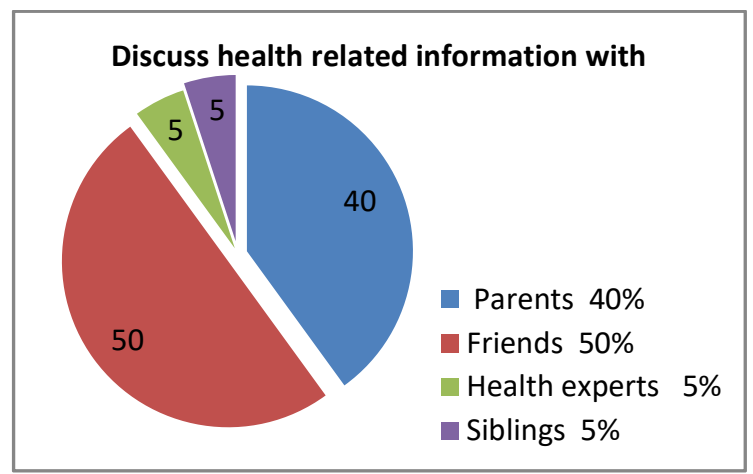

The respondents $(50 \%)$ discuss the information gathered from social media with friends whereas $40 \%$ share this information with their parents. Only $5 \%$ discuss with health experts. According to Jonathan Griffin (2018) BBC News the personal trainer or health trainers usually need insurance, an awareness of anatomy and physiology which is lacking or not mentioned on social media.

e) Deccan Chronicle (Aug, 2018) reveals that people share a large number of their photographs, information on apps and are also more concerned about their outlook which could be harmful to them. The regular distress about the appearance on social media may lead to poor health. It further discloses that women in 20s and 30s lack important minerals including potassium, magnesium and copper. Experts warn that people on social media are confused and anxious about food and unaware about what is healthy diet. The study shows that the respondents $(25 \%$ often and $70 \%$ sometimes) apply this information on themselves. 


\section{Ever applied the information on yourself}

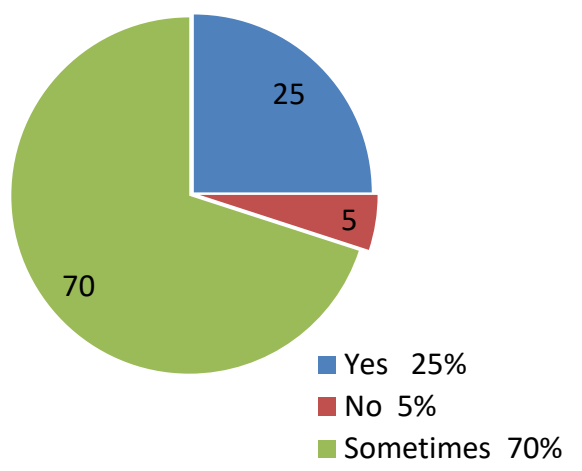

Social media is a good source of information for fitness seekers as $25 \%$ of the respondents agree that they apply and adopt the eating schedules or tips shared on social media whereas $70 \%$ of the total respondents did not deny but sometimes use this information for health fitness, fat loss etc.

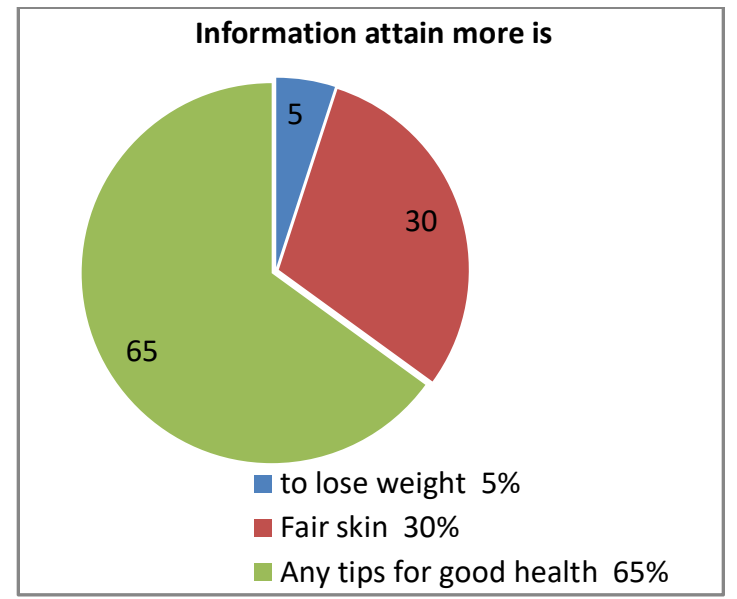

A large number of respondents (65\%) expressed that they read any tips for good health on Facebook/Instagram where as $30 \%$ of the respondents they seek more information on weight loss and 5\% dig information about fair skin.

The motive is to lose weight; wear a fair complexion or any tips for good health. Pinterest is the platform which shows information using a lot of images of fruits/fruit seeds/syrups and juices with captions projecting magic results of weight loss/weight gain attain fair skin with their application. This information is further substantiated with beautiful faces, attractive smiles recommending these magical treatments with a long list of comments. Each comment to these images adds to marketing of specified products. Most of the respondents (60\%) believe that fruits are clear diet followed by herbal plants/leaves and seeds. f) The respondents leave no stone unturned to achieve their goals of slim fits, zero figure, six pack abs, beautiful glowing faces and rejuvenating skin. This is proved as $80 \%$ of the respondents also follow the exercise video clips (30\% regularly and $50 \%$ to some extent). Unfortunately these videos are supported with huge success rate through self-applied experiences. The comments below the video are projected as the first-hand experience and rate the success persuading the other virtual friends to adapt.

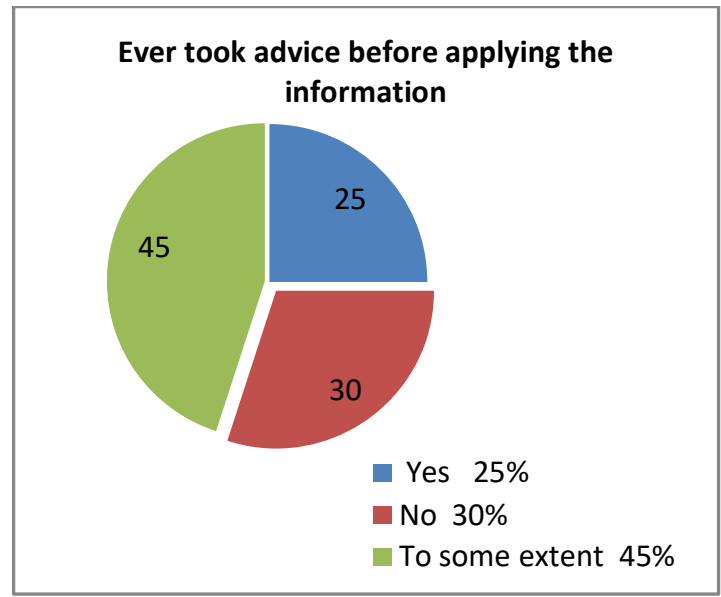

Female respondents (25\%) seek advice before applying any form of health information or tips where as $25 \%$ of the total respondents do not ask or seek any advice from experts before following It. $45 \%$ of the total respondents said that they sometimes take advice before applying the information from net. Only $5 \%$ of male respondents seek any advice related to health from experts.

g)

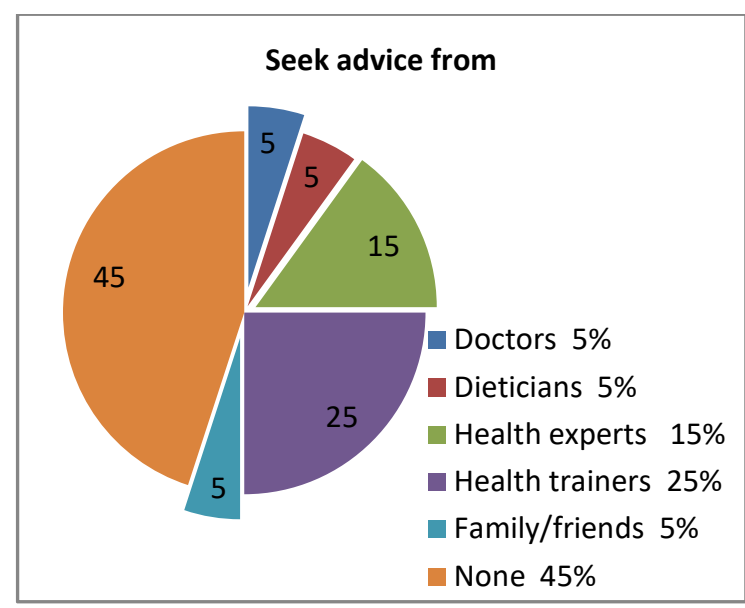

Further the data reveals that $45 \%$ respondents do not seek any advice before applying any fitness formula whereas $25 \%$ go to health trainers and only $5 \%$ seek information from family. 
h) The study also expresses that social media is a feasible tool for creating health awareness as $65 \%$ consider it a tool to spread awareness 'to some extent'. However $35 \%$ say ' $\mathrm{No}^{\prime}$ as the quarry regarding authenticity did not confirm but $65 \%$ consider it to some extent. The \%age doubt is also revealed by the respondents. $85 \%$ of the respondents express that a check is must to filter the fake information floated only for market profits. $15 \%$ of the respondent had no response 'can't say' which also explains that social media users may be ever increasing but their awareness level to check the authenticity is low.

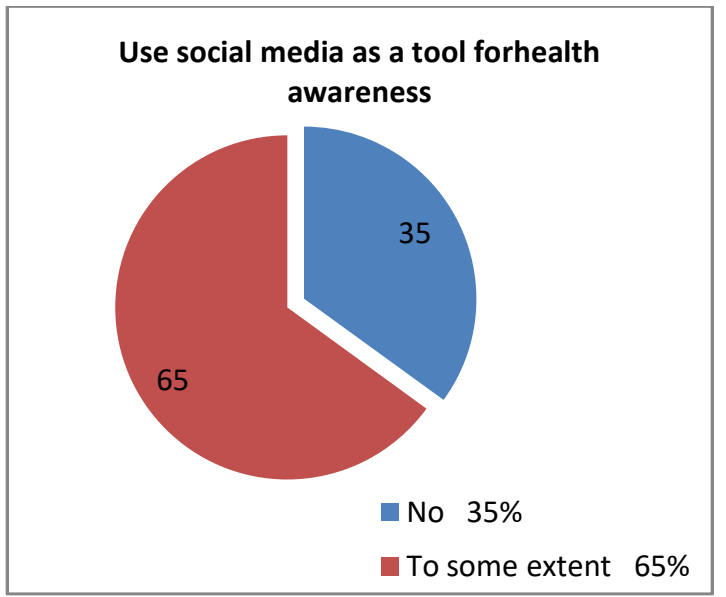

Further $35 \%$ of respondents do not use social media as a tool for health awareness whereas $65 \%$ use social media to some extent to seek health awareness.

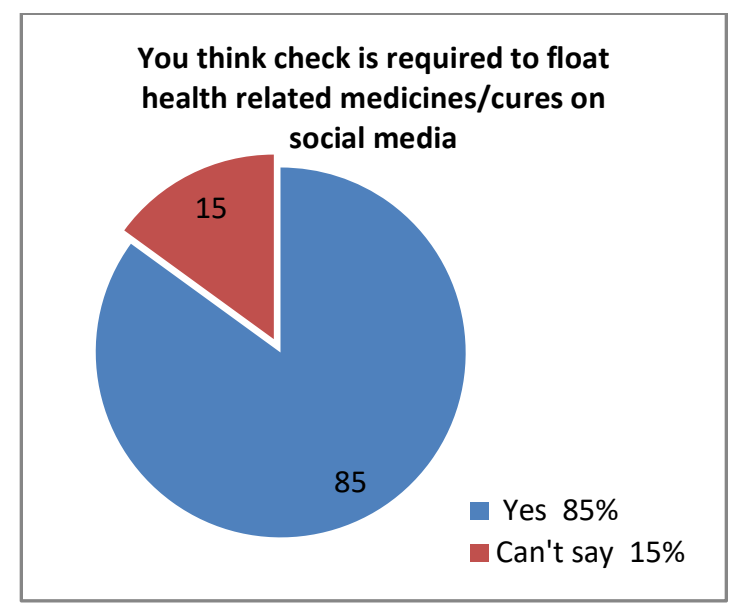

$85 \%$ of the respondents strongly support that a check is required before any health awareness information, medicines/cure are floated on social media.

i)

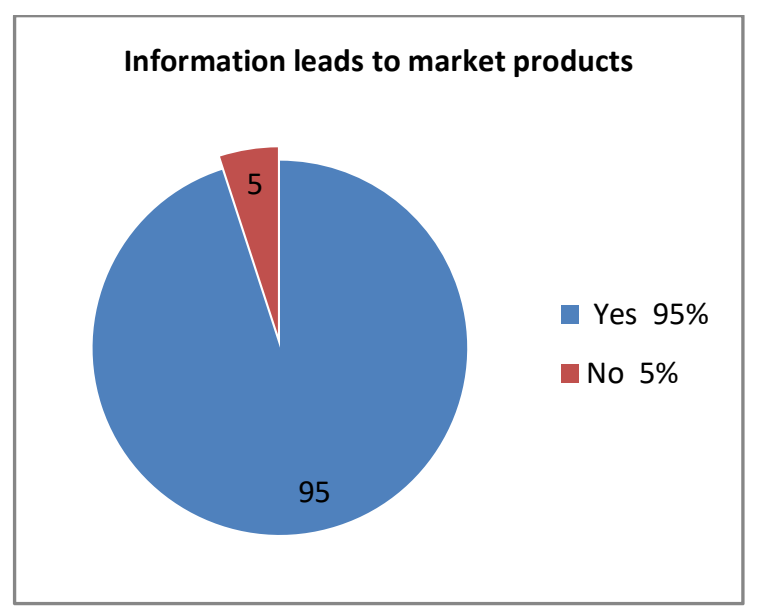

Interestingly majority of the respondents, 95\% know that almost all information related to health and fitness leads to marketing of products (medicines/supplements) for slim fit, weight loss, muscle building etc by business hubs and companies.

\section{Conclusion}

Information travels at the speed of internet. The $4 \mathrm{G}$ high speed revolution magnified the information in every field of life trivializing it practically. The social media is blurring the lines between public and private life. Common man is hardly utilizing it for the development at all levels. Though the number of social media users is incalculably multiplying but the low awareness level of the users pose a serious threat. Data generation and its consumption at bulk level do not show remarkable result in health domain. The two way communication of social media is not utilized by the individual users whereas the business hubs, marketing companies are being benefited enormously. The comments on Facebook, Instagram and video clips of 'various' recommending certain products do not only promote the products but also make the respondents as mouthpiece for the organization. Number of likes, sharing and followers also make ordinary faces as 'identified' referrals.

The portrayal of beautiful selfies, image of fair skin as 'before' and 'after' lure other virtual users for trial. The study reveals that usually people on social media blindly follow their 'friends' endangering health. The likes and comments not only substantiate the results but also create a pressure to 'do it' on others. The credibility of health trainers, experts also needs a check whereas the social media has not control to check the credentials of such experts. Even the ordinary social media users are least aware of the fake information on this platform. 
Framing of information, content and context in the field of health on social media has led agenda building where the users are becoming active players without knowing the consequences. The bulk of data on social media exists without scientific acknowledgements. Hence social media a platform to generate and apply health information is yet to develop as a tool for health communication for the health and fitness of general public. Social media with a reach around the globe with one click could prove fatal if there is no feedback and checks on the application of information in the domain of health

\section{Implications and suggestions}

The social media is a platform to generate and transfer data (health information) in bulk. The above research suggests that the health organization and the government should adopt the techniques for surveillance from where the message is generated. Social media is a tool of agenda setting and has the potential to promote health, fitness and healthy lifestyle. The study and research on the discourse of health information is the need of the hour. The feedback of the virtual audiences should be studied to meet challenges such as reliability and authenticity of the content floated on social media.

\section{References}

Ahmed, Rukhsanda\& Benjamin R. Bates. (2013) Health Communication and Mass Media: An Integrated Approach to Policy and Practice, England. Gower Publishing Limited.
BBC News (2018, October 6) Can social networks help you lose weight? Retrieved on Nov 3, 2018

Caleb, Triscari. (2018, Feb 9). Social Media influencers are shaping the popularity of 'clean eating' brands and the trend is set to continue. Retrieved on Aug 19, 2018

www.smartcompany.com.au/marketing/socialmedia/influencers-boost-healthy-eatingbrands/ Deccan Chronicle (2018, Aug 01) Women who use social media daily are likely to lack nutrition and face depressing. Retrieved on Aug 19, 2018 www.deccanchronicle.com

Holmberg, Christopher. (2014, May 05) Food and Social Media - A Complicated Relationship retrieved on Aug 19, 2018. https://huffpost.com

Manyozo, Linze. (2012). Media, Communication and Development: three approaches, New Delhi, Sage Publications India Pvt. Ltd.

Mody, Bella. (2007). Designing Messages for Development Communication: An audience participation based approach. New Delhi, Sage Publication.

Patrut, Bogdan\&Patrut, Monica. (2014) Social Media in Politics, New York, Springer

Shaikh, Javed. (2014). Communication Theory. New Delhi, Wisdom Press.

Tondon, Aditi. (2018, Oct 25) Low Carb Diet 'raises' death risk. The Tribune, pp- 1. 